\title{
ON PRIMITIVE EXTENSIONS OF RANK 3 OF SYMMETRIC GROUPS
}

\author{
TOSIRO TSUZUKU*
}

Dedicated to the memory of Professor TADAsI NAKayama

1. Let $\Omega$ be a ninite set of arbitrary elements and let $(G, \Omega)$ be a permutation group on $\Omega$. (This is also simply denoted by $G$ ). Two permutation groups $(G, \Omega)$ and $(G, \Gamma)$ are called isomorphic if there exist an isomorphism $\sigma$ of $G$ onto $H$ and a one to one mapping $\tau$ of $\Omega$ onto $\Gamma$ such that $(g(i))^{\tau}=g^{\rho}\left(i^{\tau}\right)$ for $g \in G$ and $i \in \Omega$. For a subset $\Delta$ of $\Omega$, those elements of $G$ which leave each point of $\Delta$ individually fixed form a subgroup $G_{\Delta}$ of $G$ which is called a stabilizer of $\Delta$. A subset $\Gamma$ of $\Omega$ is called an orbit of $G_{\Delta}$ if $\Gamma$ is a minimal set on which each element of $G$ induces a permutation. A permutation group $(G, \Omega)$ is called a group of rank $n$ if $G$ is transitive on $\Omega$ and the number of orbits of a stabilizer $G_{a}$ of $a \in \Omega$, is $n$. A group of rank 2 is nothing but a doubly transitive group and there exist a few results on structure of groups of rank 3 (cf. H. Wielandt [6], D. G. Higman [4]).

Now we introduce the following definition:

Definition. A permutation group $(G, \Omega)$ is an extension of rank $n$ of a permutation group $(H, \Gamma)$ if $(G, \Omega)$ is a group of rank $n$ and there exists an orbit $\Delta$ of a stabilizer $G_{a}, a \in \Omega$, such that $G_{a}$ is faithful on $\Delta$, i.e., only the identity element of $G_{a}$ induces the identity permutation on $\Delta$, and $\left(G_{a}, \Delta\right)$ is isomorphic to $(H, \Gamma)$. Moreover, if $(G, \Omega)$ is primitive (or imprimitive), it is called a primitive (or imprimitive, resp.) extension of rank $n$.

In this note we will prove the following theorem.

THEOREM. Let $S_{n}$ be the symmetric group of degree $n$.

If $S_{n}$ has a primitive extension of rank 3 , then $n=1,2,3,5$, or 7 .

2. We use the following notations:

Received May 17, 1965.

* This research was supported by the National Science Foundation, G 25213 . 
$S_{n}$ : The symmetric group of degree $n$ (on a set $\Gamma$ ).

$A_{n}$ : The alternating group of degree $n$.

$G$ : A primitive extension of rank 3 of $S_{n}$ on a set $\Omega=\{0,1,2, \ldots, n, \tilde{1}, \widetilde{2}$, $\ldots, \tilde{m}\}$ which consists of $1+n+m$ letters.

$H$ : The stabilizer $G_{0}$ of a letter, say 0 , of $\Omega$.

The orbits of $H$ are denoted by $\Delta_{0}=\{0\}, \Delta_{1}=\{1,2, \ldots, n\}$ and $\Delta_{2}=\{\tilde{1}, \tilde{2}, \ldots$, $\tilde{m}\}$ and the group $\left(H, \Delta_{1}\right)$ is isomorphic to $\left(S_{n}, \Gamma\right)$.

$L$ : The stabilizer of the subset $\{0, \tilde{1}\}$ of $\Omega$.

$\Psi$ : The character of $G$ induced by the principal character of $H$ which is called the character of the permutation representation of $(G, \Omega)$. By a well known theorem (cf. Proposition 29.2 in [6]) $\Psi$ is decomposed into three irreducible characters $\varphi_{0}, \varphi_{1}$ and $\varphi_{2}$ and one of these, say $\varphi_{0}$, is the principal character. We denote the degree of $\varphi_{i}$ by $f_{i}$. If $n \geqq 3$, then $n \neq m$ by Theorem 17.7 in [6] and so $f_{1} \neq f_{2}$ by Theorem 30.3 in [6] and we assume $f_{1}<f_{2}$.

${ }_{H} \Psi$ : The restriction to $H$ of $\Psi$. By the structure of $G,{ }_{H} \Psi$ is equal to $1_{H}+1_{s_{n-1}}^{s_{n}}+1_{L}^{S_{n}}$ where $1_{X}$ is the principal character of a group $X$ and $1_{X}^{Y}$ is the character of $Y$ induced by $1_{X}$, that is, the permutation representation of a permutation group $(Y, Y / X)$.

$$
q=(m+n+1) \cdot \frac{m \cdot n}{f_{1} \cdot f_{2}}
$$

$|X|$ : The order of a group $X$.

We use the following propositions:

Proposition 1. (W. A. Manning, Theorem 17.7 in [6]). If $n>2$, then $n<m \leqq n(n-1)$ and $m$ divides $n(n-1)$.

Proposition 2. (J. S. Frame [2]). (i) $q$ is an integer, and (ii) if $n \neq m$ then $q$ is a square.

Proposition 3. (D. G. Higman [4]). If $1+n+m=n^{2}+1$, then $n=2,3,7$ or 57 .

Let $V$ be a matrix $\left(v_{\alpha \beta}\right), \alpha, \beta \in \Omega$, of degree $1+n+m$ where

$$
v_{\alpha \beta}= \begin{cases}1 & \text { if there exists an element } g \text { of } G \text { such that } 0^{g}=\beta \text { and } \alpha \in \Delta_{1}^{g} \\ 0 & \text { otherwise. }\end{cases}
$$


Obviously, all diagonal elements of $V$ are zero and all diagonal elements of $V^{t} V$ are $n$. By calculating the traces of $V$ and $V^{t} V$ we have the following relations among $f_{1}, f_{2}, m, n$ and the eigenvalues of $V$ which are introduced by H. Wielandt (Chapter $V$ in $[6]$ ) :

Proposition 4.

$$
\begin{aligned}
& n+f_{1} s+f_{2} t=0 \\
& n^{2}+f_{1} s^{2}+f_{2} t^{2}=(m+n+1) n
\end{aligned}
$$

where $s$ and $t$ are eigenvalues of $V$ which have the multiplicities $f_{1}$ and $f_{2}$ respectively.

Proposition 5. (G. Frobenius [3]). Let $X$ be a subgroup of $S_{n}$. Then

(i) If $X$ is $S_{2} \times S_{n-2}$, then

$$
1_{X}^{s_{n}}=1_{s_{l}}+\chi^{0 \ldots 0}+\chi^{00}
$$

where $\chi^{0 \ldots 0}$ and $\chi^{00}$ are irreducible characters of $S_{n}$ (corresponding to Young diagrams $0^{0 \ldots 0}$ and ${ }^{00}$ respectively) whose degrees are $n-1$ and $\frac{n(n-3)}{2}$ respectively).

(ii) If $X$ is $S_{1} \times S_{1} \times A_{n-2}$, then

$$
\begin{aligned}
& 1_{X}^{s_{n}}=1_{s_{n}}+2 \chi^{0 \cdots 0}+\chi^{0 \cdots 0} \chi^{0 \cdots 0}
\end{aligned}
$$

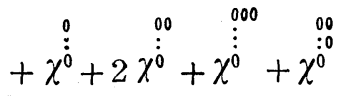

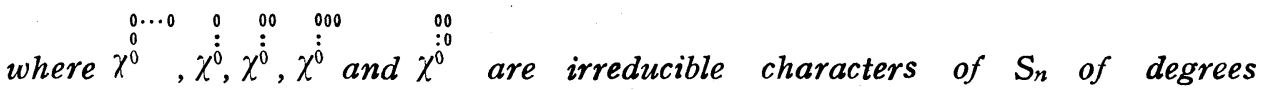
$\frac{(n-1)(n-2)}{2}, 1, n-1, \frac{(n-1)(n-2)}{2}$ and $\frac{n(n-3)}{2}$ respectively.

3. Proof of Theorem. In the following we assume that $n \neq 1,2,3,5$ and 7 . According to Proposition $1,(n-1) !>|L| \geqq(n-2)$ !.

I. The case $|L|>(n-2)$ ! and $L$ is transitive on $\Delta_{1}$.

If $L$ is a primitive subgroup of $\left(H, \Delta_{1}\right)$, then, by a theorem of $A$. Bochert (Theorem 14.2, [6]), the index of $L$ in $H$ is not less than $\left[\frac{n+1}{2}\right] !$, that is, $n(n-1)>\left[\frac{n+1}{2}\right] !$ and so we have $n=8,6$ or 4 . For those values of $n$ we know some properties of primitive subgroups of $S_{n}$ (cf. [1], $\S 166$ ). The orders 
of primitive groups of degree 8 , not containing $A_{8}$, are not divisible by 5 , but the order of $L$ is divisible by 5 . This is impossible. The orders of primitive subgroups of $S_{6}$ (or $S_{4}$ ) are divisible by 5 (or 3 resp.) and so, by Proposition 1 , the order of $L$ is divisible by 5 ! (or 3 ! resp.). This is a contradiction because $(n-1) !>|L|$. Hence $L$ is imprimitive on $\Delta_{1}$ and so there exists a non-trivial block $\Gamma$ of $\left(L, \Delta_{1}\right)$. Let $r$ be the length of $\Gamma$. Then the order of $L$ must divide $(r !)^{\frac{n}{r}}\left(\frac{n}{r}\right) !$. Therefore, by Proposition 1 , we have

$$
(n-2) ! \mid(r !)^{\frac{n}{r}}\left(\frac{n}{r}\right) ! .
$$

From this formula we have that $n=4$ or 6 . If $n=4$ then, by the assumption $(n-2) !<|L|<(n-1) !,|L|=4$ and so the degree of $(G, \Delta)$ is equal to 11 and $q=\frac{11 \cdot 4 \cdot 6}{f_{1} \cdot f_{2}}$. This is a contradiction because $q$ can not be a square for positive integers $f_{1}, f_{2}$ satisfying $f_{1}+f_{2}=10$. In the similar way, for the case $n=6$, we have $q=\frac{22 \cdot 6 \cdot 15}{f_{1} \cdot f_{2}}$ or $\frac{17 \cdot 6 \cdot 10}{f_{1} \cdot f_{2}}$ which also show us contradictions.

II. The case $|L|>(n-2)$ ! and $L$ is intransitive on $\Delta_{1}$.

Since $L$ is a subgroup of $S_{r} \times S_{n-r}$ with a positive integer $r$, we have the relation $(n-2) ! \mp r !(n-r)$ ! Hence we have the following cases (we assume $r \leqq n-r): r=1$ or 2 .

(i) $r=1$ : Since $L \subseteq S_{1} \times S_{n-1}$ and $(n-1) !>|L|>(n-2)$ !, $L$ must be $S_{1} \times A_{n-1}$. Now we take up an element $\sigma_{0}$ of $H$ which is a cycle of length 3 as an element of $\left(H, \Delta_{1}\right)$. Then we see that, as an element of $\left(H, \Delta_{2}\right), \sigma_{0}$ is the product of disjoint two cycles of length 3. Therefore $\sigma_{0}$ is the product of disjoint three cycles of length 3 and $\Psi(\sigma)=3 n-8$. Let $\sigma$ be an element of $H$ which is the product of disjoint $r$ cycles of length 3 as an element of $\left(H, \Delta_{1}\right)$. Then, in the similar manner, $\sigma$ is the product of exactly disjoint $3 r$ cycles of length 3 . This concludes that if an element $\sigma$ of $H$ is conjugate to $\sigma_{0}$ in $G$ then they are conjugate in $H$ already. Hence the number of elements which are conjugate to $\sigma_{0}$ is

$[G: H] \cdot$ the number of elements of $H$ which are conjugate to $\sigma_{0} / \Psi(\sigma)$

$$
\begin{aligned}
& =\frac{(3 n+1) \cdot n !}{(3 n-8) \cdot 3 \cdot(n-3) !} \\
& =\frac{(3 n+1) n(n-1)(n-2)}{3(3 n-8)}
\end{aligned}
$$


Since this number is an integer, $3 n-8$ must divide $8 \cdot 5 \cdot 2$ and this concludes $n=16,8,6$ or 4 . If $n=16$, then, in the similar manner, the number of elements of $G$ which are conjugate to an elements $\sigma_{1}$ of $H$ which is a cycle of length 5 as an element of $\left(H, \Delta_{1}\right)$ is equal to $\frac{49 \cdot 16 !}{34 \cdot 5 \cdot 11 !}$ and, since this number is not an integer, we have a contradiction. If $n=8$, then the number of elements of $G$ which are conjugate to an element $\sigma_{2}$ of $H$ which is the product of disjoint two cycles of length 2 as an elements of $\left(H, \Delta_{1}\right)$ is equal to $\frac{25 \cdot 8 !}{13 \cdot 2^{2} \cdot 2 \cdot 4 !}$ and, since this number is not an integer, we have a contradiction. If $n$ is either 6 or 4 , the degree of $(G, \Omega)$ is a prime number and so, by theorems of Galois (Theorem 11.6 in $[6]$ ) and Burnside (Theorem 11.7 in $[6]),(G, \Omega)$ is a Frobenius group. This is a contradiction.

(ii) $r=2$ : Since $L$ is a subgroup of $S_{2} \times S_{n-2}$ and $(n-1) !>|L|>(n-2)$ !, $L$ must be $S_{2} \times S_{n-2}$. Then $H^{\Psi}=31_{s_{n}}+2 \chi^{0 \cdots 0}+\chi^{00}$ and so we have the following possibilities

$$
\begin{array}{llc}
f_{1}=n & & 2 n-1 \\
f_{2}=\frac{n(n-1)}{2} & & (n-1)(n-2) \\
2
\end{array}
$$

In the first case, according to Proposition 3, we have

$$
\begin{gathered}
n+s n+\frac{\operatorname{tn}(n-1)}{2}=0 \\
n^{2}+s^{2} n+\frac{t^{2} n(n-1)}{2}=\frac{n\left(n^{2}+n+2\right)}{2}
\end{gathered}
$$

and so $n=\frac{t^{2}+4 t}{2-t^{2}}$, that is, $n$ is 2 or 5 which has been excluded. In the second case we have

$$
q=\frac{n^{2}+n+2}{2} \times \frac{n^{2}(n-1)}{2(n-1) \cdot \frac{(n-1)(n-2)}{2}}=\frac{n^{2}\left(n^{2}+n+2\right)}{2(2 n-1)(n-2)},
$$

but this is not a square for any integer $n$. This is a contradiction, by Proposition 2 .

III. The case $|L|=(n-2)$ !. Then $m=n(n-1)$ and so the degree of $(G$, $\Omega$ ) is $n^{2}+1$. By Proposition 3, $\boldsymbol{n}=57 . \quad m=57 \cdot 56=3192$ and so $q=\frac{3250 \cdot 57 \cdot 3192}{f_{1} \cdot f_{2}}$ must be a square. Then we have the following possibilities: 


$$
\begin{array}{lll}
f_{1}=624 & & 1520 \\
f_{2}=2625 & \text { or } & \\
& & 1729 .
\end{array}
$$

On the other hand, since $L$ is intransitive and since $|L|=55$ !, $L=S_{1} \times S_{1} \times S_{55}$ or $L=S_{2} \times A_{55}$ or $L=$ the group which consists of even permutations in $S_{2} \times S_{55}$. In any of those cases, since $1_{S_{1} \times S_{1} \times A_{65}}^{S_{67}}=1_{L}^{S_{67}}+$ a sum of characters of $S_{67}$ and since $1+1_{S_{66}}^{S_{67}}+1_{S_{1} \times S_{1} \times A_{65}}^{S_{67}}$ is decomposed into 13 irreducible characters which have degrees $1,1,1,1,56,56,56,56,56,57 \cdot 27,57 \cdot 27,28 \cdot 55$ and $28 \cdot 55$ respectively, $f_{1}$ and $f_{2}$ must be partial sums of these integers, but it is impossible.

Thus we complete the proof of Theorem.

4. There exist primitive extensions of rank 3 of $S_{n}$ for $n=1,2,3,5$ and 7 .

(i) The cyclic group of order 3 is the unique primitive extension of $S_{1}$.

(ii) The dihedral group of order 10 is the unique primitive extension of $\mathrm{S}_{2}$

(iii) The alternating group $A_{5}$ of degree 5 is the unique primitive extension of $S_{3}$.

(iv) Let $N$ be the elementary abelian group of order 16 and let $\boldsymbol{a}_{1}, \boldsymbol{a}_{2}, \boldsymbol{a}_{3}$, $a_{4}$, be a minimal set of generators of $N$. For any element $\sigma$ of $S_{\overline{5}}$ a permuta-

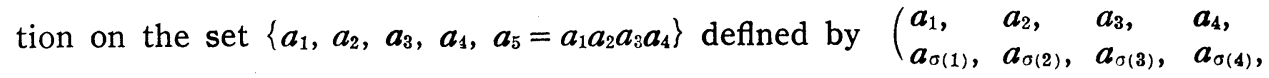
$\left.\begin{array}{l}a_{5} \\ a_{\sigma(5)}\end{array}\right)$ induces an automorphism $\bar{\sigma}$ of $N$. Thus $S_{5}$ is identified with an automorphism group $H$ of $N$. Then we can see easily that the semidirect product $S_{5} N$ is the unique primitive extension of rank 3 of $S_{5}$.

(v) Let $F$ be the finite filed consisting of $5^{2}$ elements and let $\sigma$ be the involutive automorphism of $F$ and let $U_{3}(F)$ be the projective special unitary group over $F$ of dimension 3. Then $\sigma$ induces an automorphism $\bar{\sigma}$ of $U_{3}(F)$. $U_{3}(F)$ contains a $\bar{\sigma}$ invariant subgroup $H$ which is isomorphic to $A_{7}$ and the semidirect product $\langle\bar{\sigma}\rangle H$ of groups $\langle\sigma\rangle$, which is generated by $\bar{\sigma}$, and $H$ is isomorphic to $S_{7}$ (H. H. Mitchell; Theorem 25, [5]). $U_{3}(F)$ is a primitive extension of rank 3 of $A_{7}$ (D. G. Higman [4]). Then the semidirect product $\langle\bar{\sigma}\rangle U_{3}(F)$ is a primitive extension of $\operatorname{rank} 3$ of $\langle\bar{\sigma}\rangle H \cong S_{7}$.

\section{REFERENCES}

[1] W. Burnside, Theory of groups of finite order, 2nd ed. Cambridge University Press, London, 1911. 
[2] J. S. Frame, The double cosets of a finite group, Bull. Amer. Math. Soc., 47 (1941), 458-467.

[3] G. Frobenius, (i) Über die Charakter der symmetrishen Gruppe, Sitzber. Preuss. Akad., Berlin (1900), and, (ii) Über die Charaktere der alternierenden Gruppe, ibid. (1901), 303-315.

[4] D. G. Higman, Finite permutation group of rank 3, Math. Zeitshr. 86 (1964), 145-156.

[5] H. H. Mitchell, Determination of the ordinary and modular ternary linear groups, Trans. Amer. Math. Soc. 12 (1911), 207-242.

[6] H. Wielandt, Finite permutation groups, Academic Press, New York and London, 1964.

Nagoya University and

University of Illinois 